\title{
Comunicar emociones en el discurso metapolítico de twitter: el caso de \#MADURO versus @NICOLASMADURO
}

\section{Communicating emotions in the metapolitical discourse of twitter: the case of \#MADURO versus @NICOLASMADURO}

\author{
* University of Navarra, Spain
}

Ana Maria Fernandez Vallejo*

Resumen

\begin{abstract}
Redes sociales, como Twitter o Facebook, están permitiendo un espacio esencial para el desarrollo del debate político. En este estudio, investigamos cómo se comunican las emociones en este entorno público prestando una especial atención a qué emociones emergen, qué estimulo las provoca, y con qué objetivo y de qué modo se comunican. Se analiza un corpus de 1353 tuits, que contiene \#Maduro (Subcorpus1) o @NicolasMaduro (Subcorpus2). Estos tuits se publicaron el 24 de julio de 2017, fecha equidistante entre el 16 de julio, día en que la oposición a Maduro convoca un referéndum y, el 30 julio, día de la votación oficial para la Asamblea Constituyente. A través de la lectura directa de tuits y combinando el método cuantitativo y cualitativo, demostramos que las emociones son una parte esencial de esta red $y$, entre ellas, las más frecuentes son 'ira' y 'temor'. Asimismo, el análisis reveló que este corpus, claramente polarizado, reúne más tuits en contra de Maduro que a favor. Respecto a las funciones, cabe destacar cómo Twitter se convierte en una herramienta persuasiva de campaña, con dos funciones clave 'criticar' o 'apoya' a Maduro. Finalmente, el análisis formal arroja un mayor número de expresiones emocionales negativas (léxico emocional, trolling e insultos).
\end{abstract}

Palabras clave: Emociones, Redes sociales, Opinión pública, Venezuela.

Abstract

Social networks, such as Twitter or Facebook, are providing an essential space for the development of political debate. In this study, we investigate how the emotions are communicated in this public sphere, paying special attention to emotions, stimulus, purpose and style. We analyse a corpus of 1353 tweets, which contain \#Maduro (Subcorpus1) or @NicolasMaduro (Subcorpus2). These tweets were published on July 24, 2017, a date between July 16 -when the opposition to Maduro convened a referendum- and, on July 30 -the day of the official vote for the Constituent Assembly. Through the direct reading of tweets and using a combination of quantitative and qualitative methods, we demonstrate that the emotions are an essential part of this network and, among them, the most frequent are anger and fear. Additionally, the analysis has revealed that this corpus exhibits a high polarization between the supporters and the opponents of Maduro, yet Maduro's opponents' tweets prevail. In order to understand the function of these tweets, our findings show that citizens use Twitter as a persuasive campaign tool to criticize Maduro or, alternatively, to support him. Finally, regarding expression, the formal analysis conducted notes how the negative expression (emotional, trolling, insults) prevails.

Keywords: Emotions, Social Media, Public Opinion, Venezuela.

\section{Introducción}

Con la red, el mundo de la política, el activismo y las democracias han cambiado. Ya no solo se oye a los políticos; el ciudadano de a pie, gracias a los nuevos medios sociales, puede expresarse con libertad y sabiendo que su voz llegará lejos, a audiencias inimaginables. Las nuevas tecnologías de la información, tal y como ya hace más de veinte años admitieran Rheingold (1993) y Grossman (1995), han servido para crear una democracia directa que acote la distancia entre gobernados y gobierno, y donde el ciudadano se expresa 
de forma directa sin necesidad de formar parte de las actividades tradicionales de la política -los partidos políticos o instituciones-. En unos casos, intentará reforzar los poderes establecidos; en otros, movilizar y activar fuerzas opositoras para que luchen contra los poderes creados, especialmente cuando estos gobiernos manifiestan posturas intransigentes (Bonilla and Rosa, 2015; Castells, 2012).

Es un hecho que las redes sociales, Twitter y Facebook principalmente, han desempeñado un papel fundamental en las protestas civiles modernas. Su fácil acceso y uso les ha permitido un protagonismo extraordinario en las movilizaciones sociales, donde el ciudadano se ha visto empoderado. Al respecto, cabe recordar la influencia e impactos logrados en distintos lugares del mundo: la "Primavera árabe" en el Norte de África, primero en Túnez (Castells, 2012; De León, 2011), seguido por Egipto (Aourag \& Alexander, 2011; Borge-Holthoefer et al., 2015) y, después, en Libia y Siria (Garwood-Gowers, 2013). En 2011 en España, surge el movimiento del 15M (Castells, 2009; 2012), al que sigue la protesta norteamericana en septiembre de 2011, es el denominado "Occupy Wall Street", \#occupywallstreet (Constanza-Chock 2012; Tarrow 2011) y, en 2012, la "Primavera mexicana", con el movimiento \#YoSoy132 (Nabel, 2015). Por tanto, parecen existir evidencias para creer en el sueño de la "democracia virtual" a partir de nuevas relaciones horizontales y debates políticos que propician las redes digitales.

El punto de vista seleccionado para este estudio será el emocional. Las emociones son esenciales para entender las actitudes y comportamientos en el terreno político (Zajonc, 1980). El estudio de las emociones dentro del ámbito político es relativamente reciente, pero ha ido ganando terreno de año en año (Albertson and Gadarian 2015; Marcus et al. 2000; Valentino et al. 2011; Zeitzoff 2016), del mismo modo que ha ocurrido en otros ámbitos, como el marketiniano (Stieglitz y Dang-Xuan, 2013), el sociológico (Dodds et al., 2011), el económico (O'Connor et al., 2010) o lingüístico (Lee y Kahle, 2016).

Las emociones en los primeros momentos eran vistas como perjudiciales para el buen gobierno (Kuklinski et al., 1991); sin embargo, estudios posteriores han demostrado que las emociones son definitivas en la vida política: en la participación política (Valentino et al., 2011), en la opinión pública (Brader and Corrigan, 2006; Grillo, 2017; Huddy et al. 2008), en el procesamiento de la información política (Lodge and Taber, 2005), en la discusión política (Tam Cho et al., 2013), en la actitud ante la política (Albertson and Gadarian, 2015) y en la comunicación de la política (Hutchings eta al. 2006). Aquellos que han estudiado las emociones en política se han centrado, principalmente, en el impacto de las distintas emociones en el lenguaje político de las campañas y de los dirigentes políticos (Brader and Corrigan, 2006; Bruns and Burgess, 2011; Graham et al., 2013; Vergeer et al., 2013). Sin embargo, este artículo se centra en el discurso metapolítico; el objetivo principal es explorar cómo el ciudadano reacciona emocionalmente en momentos de campaña electoral elecciones, puesto que los estudios realizados hasta este momento en este campo han sido modestos (Dang-Xuan et al., 2013; Hosch-Dayican et al., 2016).

Este artículo fija su atención en Venezuela, en el verano de 2017, en los tuits publicados el 24 de julio a raíz de la situación política que vive el país. El momento seleccionado es de vital importancia para la historia venezolana y se enmarca entre la convocatoria de los comicios celebrados entre el 16 y 30 de julio de 2017 en Venezuela, dos votaciones de características legales y políticas muy distintas. Las elecciones, piedra angular de cualquier sistema democrático, preocupan a los ciudadanos de un país. Sin embargo, cuando estas elecciones se convocan en situaciones complicadas desde el punto de vista económico, político y social -o incluso legal-, como es el caso de Venezuela, su resultado se vuelve aún más transcendente, y la 
resonancia en los medios sociales experimenta un incremento considerable, tal y como lo explica HoschDayican (2014) y Mossberger et al. (2008).

El análisis así planteado y centrándose en el caso de las elecciones la Venezuela de julio de 2017 desea responder a los siguientes objetivos:

1. Evaluar si los mensajes de Twitter reflejan las emociones de los ciudadanos en torno a los temas político tratados.

2. Precisar qué emociones prevalecen en los tuits de los ciudadanos en torno a los temas políticos debatidos.

3. Identificar cuál es la causa de la emoción.

4. Reconocer con qué función se publica el tuit emocional.

5. Determinar qué recursos verbales y no verbales ayudan a expresar las emociones y su intensidad.

\section{Presente estudio: estado de la cuestión, metodología y corpus}

Estado de la cuestión: emociones, redes sociales y comunicación

De acuerdo con los objetivos planteados, el primer paso de esta investigación era estudiar el estado de la cuestión sobre las emociones. Se han realizado importantes esfuerzos a la hora de definir qué es una emoción; pero no se ha llegado a una respuesta unánime (Brosch et al., 2013; Scheff, 2015). Sin embargo, conviene partir, al menos, de una definición de trabajos que nos permita entender a qué realidad nos estamos refiriendo. La propia etimología de la palabra puede ayudar. El étimo 'e-moción' procede de la palabra latina "movere"; por tanto, se puede considerar que las emociones son los movimientos internos (del 'alma') de cada sujeto ante estímulos externos específicos (Damasio, 1999; Russell, 2009). Estas reacciones ante el entorno son subjetivas y alcanzan un espectro muy amplio que varía en términos de estímulo -intensidad- y valencia -positiva o negativa- (Scherer et al., 2013). Más específicamente en el contexto político, pensamos en una emoción como el resultado de un proceso por el cual los individuos evalúan el significado de una situación o decisión política, y actúan acorde a su modo de percibirla (Marcus, 2003; Smith y Ellsworth, 1985). Por ejemplo, ante un resultado electoral un individuo puede sentir sorpresa; otros, ansiedad; otros, felicidad.

Para determinar qué emociones aparecían en los tuits, se precisaba disponer de una clasificación válida, precisa y flexible, puesto que tampoco existe un consenso en torno a la taxonomía de las emociones (Scheff, 2015). Se identifican 9 emociones, adaptando la rueda emocional propuesta por el grupo Grid (Sherer et al., 2013): con valencia positiva se distinguen 'alegría', 'orgullo', 'confianza', 'deseo'; y con valencia negativa, 'tristeza', 'vergüenza', 'ira', 'temor', 'sorpresa'. Se establecen pares de emociones opuestas, así a cada emoción se le asigna un número y un signo positivo (+) o negativo (-). Y si esa emoción aparece con una intensidad mayor se le añade dos signos, tal y como se puede ver en Figura 1. La definición de cada una de estas emociones genera de nuevo problemas a los expertos; sin embargo, la Erro! A origem da referência não foi encontrada., propuesta por Scheff (2015), puede clarificar qué estimulo provoca alguna de ellas y cómo nuestro cuerpo se prepara físicamente para distintas reacciones, según la emoción. 
Tabla 1: Emociones según Scheff (2015)

\begin{tabular}{lll}
\hline Emoción & Estímulo & Preparación para \\
\hline Tristeza & pérdida & llorar \\
\hline Temor & peligro físico & temblar y sudar \\
\hline Ira & frustración & acalorarse \\
\hline Orgullo & sentimiento de aceptación & no preparación \\
\hline Vergüenza & sentimiento de rechazo & "risa nerviosa" \\
\hline
\end{tabular}

Figura 1: Clasificación de las emociones

En un primer momento se identificó el comportamiento que cada publicación mantiene con respecto a las emociones (Tabla 4). Se distingue entre tuits neutros emocionalmente y tuits emocionales. Al respecto, es necesario aclarar que no solo se considerarán tuits emocionales aquellos que describan una emoción, a través del llamado léxico emocional (ej.: "me siento feliz") (Foolen, 2011), sino también aquellos que provocan una emoción en el lector (ej.: "tiene cáncer"), a través de las denominadas "emotion-laden words" (Braun, 2015). Por tanto, asumimos que la emoción puede ser razón para la actividad comunicativa y, al revés, que el acto comunicativo puede provocar la emoción. Estudios similares ya se habían realizado en el ámbito marketiniano (Strapparava and Milhacea, 2007) o en la política (Mohammad et al., 2015). Mohammad et al (2015), a partir del análisis de la campaña americana de 2012, había concluido que un 87,98 \% son tuits emocionales. Este estudio nos permite formular la hipótesis 1 del siguiente modo:

H1. Hay un mayor número de tuits emocionales que informativos en el corpus seleccionado. Por su parte, Brader y Corrigan (2006) habían realizado un análisis en torno a qué emociones estaban presentes en los anuncios televisivos de la campaña americana de 2000 y los resultados obtenidos indicaban que las emociones con más presencia eran 'ira', 'temor', 'deseo' y 'orgullo'. De este modo enunciamos la hipótesis 2.

H2. Las emociones con una frecuencia más alta serán 'ira', 'temor', 'deseo' y 'orgullo'. A continuación, se discute la emoción en función de qué hecho o persona provoca el estado emocional, es el denominado "estímulo", según la teoría de los llamados "roles semánticos". Una teoría con gran tradición en la lingüística (Gildea and Jurafsky, 2002), que se ha reutilizado para los estudios relacionados con las 
opiniones de productos, así como el análisis de las emociones (Baker et al., 1987; Mohammad et al., 2015). Puesto que el corpus seleccionado tomaba como criterio de búsqueda \#Maduro o @NicolasMaduro, cabía esperar que el estímulo era Maduro; por ello, esta fase del análisis (Figura 2) estudiaba la polarización de los tuits emocionales seleccionados teniendo en cuenta la figura de Maduro (Morales et al., 2015). Lassen and Brown (2011) encontraron que el electorado tiende a participar más, especialmente cuando son miembros de grupos minoritarios. Por tanto, podríamos enunciar la hipótesis 3 del siguiente modo:

H3. Los tuits contrarios a Maduro estarán en mayor proporción que los tuits de los seguidores favorables a Maduro.

En tercer lugar, se estudia qué objetivo final subyace en los tuits emocionales publicados, de acuerdo con la propuesta de Mohammad (2015) y Persson (2017); estos se habían inspirado en trabajos anteriores realizados desde esta perspectiva, pero aplicados en áreas distintas a la política: Collier et al. (2011), en el ámbito sanitario; y Caragea et al. (2011), en el ámbito social. En este sentido se clasificaron los tuits de acuerdo con las siguientes funciones: 'admirar', 'amenazar', 'apoyar', 'denunciar', 'desahogarse', 'incitar a la acción', 'informar', 'insultar', 'mostrar desacuerdo', 'mostrar hipocresía', 'provocar' y 'ridiculizar' (Tabla 6). Los resultados obtenidos en trabajos como el de Mohammad (2015), quien había encontrado que los objetivos con más presencia eran 'apoyar', 'admirar', 'desahogarse' y 'mostrar hipocresía', nos llevan a plantear la hipótesis 4 en los siguientes términos:

H4. Las funciones 'apoyar', 'admirar', 'desahogarse' y 'mostrar hipocresía' son las que más presencia tienen en los tuits publicados.

En última instancia, nos centramos en estudiar cómo se comunican esas emociones ( Tabla 7); son las llamadas estrategias de evaluación e intensificación, siguiendo la terminología de Caffi y Janey (1994). Se analizan las elecciones verbales y no verbales que sugieren una expresión emocional o ayudan a intensificarla (Bednarek, 2008; Foolen, 2011; Guerrero, 2014). Se tendrá en cuenta el léxico emocional y "emotion-laden words" (Foolen, 2012: 341-2), y otros tipos de recursos, especialmente estilísticos, que pueden ser considerados como indicios de emocionalidad: insultos y posts denominados "trolling"; ironía y preguntas retóricas (Gibbs, 2000; Kreuz, 1996); emoticonos (Go et al., 2009), repetición de letras y signos, y uso de mayúsculas no normativo (Brody and Diakopoulos, 2011).

Cabe destacar dentro de este análisis, los recursos no verbales propios del lenguaje mediatizado por ordenador: emoticonos y repetición de letras y signos de exclamación, así como mayúsculas, usadas al margen de la norma. El lenguaje de las redes es un híbrido entre el discurso oral y escrito, y el emisor intenta recrear el lenguaje gestual y corporal propio de la lengua hablada a través de iconos y símbolos, que de una manera o de otra ayudan a interpretar el correcto enfoque emocional del mensaje (Calero Vaquera and Vigara Tauste 2014). Otro rasgo exclusivo de este lenguaje son los denominados "trolling", mensajes agresivos y maliciosos con un tono bronco y palabras gruesas y, en muchos casos, escenas obscenas cuyo objetivo fundamental es provocar emocionalmente a los receptores e incendiar el conflicto planteado (Hopkinson, 2013). 


\section{Corpus y metodología}

Para abordar el análisis planteado, se recogieron tuits los días 20, 22, 24, 26, 28 y 29 de julio de 2017, donde aparecía el hahstag \#Maduro o la mención @NicolasMaduro (Tabla 2), organizados en dos subcorpus: C1 (\#Maduro) y C2 (@NicolasMaduro). Con este hashtag y mención nos asegurábamos de que el tuit era relevante para la política de Venezuela en un momento de gran tensión en el gobierno de Nicolás Maduro. Aunque ambas herramientas contribuyen en el grado de influencia y expansión de los mensajes de los tuits (Cha et al., 2010), cada una de ellas lo hace de un modo distinto desde el punto de vista social y lingüístico (Persson, 2017). La mención equivale a un vocativo dentro del tuit (Zappavigna, 2012) y la cuenta aludida recibe automáticamente un aviso en la función 'notificaciones' de que ha sido mencionada; por tanto, hay una alusión directa a una audiencia concreta. Por su parte, el hashtag se comporta como una mera etiqueta facilitadora de conversaciones (Rossi and Magnani, 2012) o discusiones (Bruns and Burgess, 2011), al mismo tiempo que genera temas fácilmente localizables en el ecosistema de Twitter (Persson, 2017). Por tanto, parece lógico pensar que el comportamiento emocional de ambos subcorpus sería distinto y así enunciamos las hipótesis 5 y 6 :

H5. En las publicaciones con el hashtag \#Maduro prevalecen las emociones negativas.

H6. En las publicaciones con la mención @NicolasMaduro prevalecen las emociones positivas.

Para entender el momento político en el que se inscriben los tuits recogidos conviene recordar brevemente qué ocurren los domingos 16 y 30 de julio de 2017. Empezaremos por la segunda fecha. El 30 de julio se celebraron las elecciones que Maduro había convocado para elegir la Asamblea Constituyente, formada por 545 miembros, todos ellos leales al presidente Maduro, y encargada de redactar una nueva constitución para el país. Sin embargo, desde que Nicolás Maduro toma esta decisión, el 1 de mayo de ese año, la coalición opositora, la llamada Mesa de la Unidad Democrática (MUD) entiende que se trataba de una medida que ponía en peligro directamente la democracia (Iriarte, 2017). Por ello, dos semanas antes de estas elecciones, el 16 de julio, la oposición celebra un referéndum para rechazar la Constituyente (Meza, 2017). Cabe destacar, por tanto, que el sistema político venezolano está absolutamente polarizado entre los partidarios del candidato Maduro y la coalición opositora.

Los tuits fueron extraídos a través del programa de analítica Twitter Twitonomy, al que se accedió a la misma hora dichos días y que arrojaba un total de 3000 tuits por búsqueda. Se dispone de un total de 24000 tuits. Un corpus tan amplio parecía obligar a un análisis de emociones automatizado, tal y como ya se había realizado en otros estudios con la ayuda de nuevas aplicaciones (Dodds and Danforth, 2010; Zhai and Liu. 2005) y/o algoritmos (Liu, 2012; Mohammad, 2015; Pang and Lee, 2008; Vilares and Alonso, 2016). Sin embargo, se decide evitar este tipo de análisis automatizado y se opta por una lectura directa de tuits, que nos obliga, por una parte, a reducir la muestra de análisis, lo que significa una limitación en la investigación, pero por otra nos ofrece una aproximación más exacta y profunda de los textos. Este estudio directo permite interpretar fenómenos comunicativos como ironías, lenguaje figurado o sarcástico, que en el análisis de grandes corpus sigue generando problemas todavía sin resolver (Ghosh et al., 2015; Grimmer and Stewart, 2013). 
De los tuits recogidos, se opta por analizar un solo día, y se toma como referencia el 24 de julio de 2017, fecha equidistante entre el 16 de julio, día en que la oposición convocó a las urnas, y el 30 julio, día de la votación oficial para la Constituyente. Se partía de un total de 6014 tuits, de los que se desestimó 4751 retuits. Finalmente el corpus analizado queda conformado por 1353 tuits: 620 tuits, pertenecientes a C1 y 733, a C2, tal y como se puede ver en Tabla 2.

Tabla 2: Tuits por corpus

\begin{tabular}{lllllll}
\hline & \multicolumn{2}{l}{ Corpus (24 julio) } & \multicolumn{2}{l}{ C2: @NicolasMaduro } & \multicolumn{2}{l}{ C1: \#Maduro } \\
& Tuits & Palabras & No tuits & Palabras & N0 tuits & Palabras \\
\hline Corpus inicial & 6104 & 105309 & 3034 & 55572 & 3070 & 49737 \\
\hline Retuits & 4751 & 85415 & 2414 & 45880 & 2337 & 39535 \\
\hline Corpus final & 1353 & 19894 & 620 & 9692 & 733 & 10202 \\
\hline
\end{tabular}

Para determinar el comportamiento emocional de los tuits seleccionados, se crea un cuestionario. Un anotador lee el tuit y responde, en primer lugar, a la pregunta: ¿El tuit presenta una actitud emocional? Solamente si el tuit es considerado emocional, se sigue con el resto del cuestionario, cuyas preguntas son las siguientes:

1. ¿Cuál de las siguientes actitudes emocionales prevalece?

\begin{tabular}{lll} 
- Alegría & - Tristeza \\
- Orgullo & - & Vergüenza \\
- Deseo & - & Ira \\
- & - Temor \\
\hline
\end{tabular}

2. ¿Esta emoción es a favor o en contra de Maduro?

3. ¿Cuál de las siguientes funciones describe mejor el fin del tuit?

- Denunciar

- Informar

- Ridiculizar

- Apoyar

- Insultar

- Admirar

- Mostrar hipocresía

- Amenazar

- Incitar a la acción

- Provocar

- Desahogarse

- Mostrar desacuerdo

4. ¿Cuál de los siguientes recursos ayudan a expresar mejor la emoción?

- Léxico emocional

- Insultos

- Trolling

- Ironía

- Preguntas retóricas

- Emoticonos

- Repetición de mayúsculas

- Repetición de signos (i, !!, i!!!, i!!!!)

Veamos a continuación la Tabla 3, para entender cómo se ha analizado cada uno de los tuits tomando como ejemplo el tuit siguiente: "@username @username @username @NicolasMaduro Deseos no empreñan. LA CONSTITUYENTE SI VA! OH EH OH EH OH S $_{\text {" }}$ 
Tabla 3: Criterios de análisis

\begin{tabular}{lll}
\hline Criterio de análisis & Resultado & \\
\hline Emoción & Alegría & $(\S 3.1)$ \\
\hline Estímulo (polarización) & Maduro (a favor) & $(\S 3.2)$ \\
\hline Función & Apoyar & $(\S 3.3)$ \\
\hline Expresión de la emoción & Mayúsculas, repetición de sonidos & $(\S 3.4)$ \\
\hline
\end{tabular}

Para comprobar la validez del cuestionario se realiza un pretest aplicando, en primer lugar, el cuestionario a 100 tuits del día seleccionado. Asimismo para comprobar la fiabilidad del método, se pide la respuesta también a un segundo anotador quien evalúa el 10\% de los tuits (135 tuits). Las coincidencias entre los dos anotadores que realizaron la tarea fueron superiores al $85 \%$ en cada pregunta del cuestionario. Una vez se aprueba el diseño del cuestionario, se decide aplicar el mismo cuestionario a una muestra de 500 tuits, extraída uniformemente de los días 20, 22, 26, 28 y 29. Los resultados obtenidos arrojan una coincidencia media de $75.3 \%$, que nos permiten extrapolar los datos obtenidos y asegurar la consistencia de la muestra planteada, siempre con las reservas pertinentes.

Por tanto, analizamos los tuits emocionales combinando el análisis cualitativo y cuantitativo. Una lectura directa de los dos subcorpus nos permite la cuantificación y codificación de lo tuits en función de las emociones encontradas (Tabla 4 y Tabla 5), su comportamiento con respecto a Maduro (Figura 2) y la función con que se publica (Tabla 6).

Para detectar el léxico emocional, se crea una lista de palabras con su frecuencia usando el programa Sketch Engine (Kilgarriff et al., 2014). A continuación, se eliminan stop words, adverbios, y los sustantivos y adjetivos restantes se cotejan con el lexicón ML-SentiCon ${ }^{1}$, uno de los pocos existentes en castellano (Cruz et al., 2014). Dicho léxico está conformado por 8 capas y cada una de las capas por lemas que presentan una polaridad emocional positiva o negativa y un grado de intensidad de entre -1 y 1 . El cotejo, realizado con la ayuda de Excel, solo se implementa con los lemas que ML-SentiCon recoge en las capa 1 y 2 , que son las que presentan un mayor grado de fiabilidad (Cruz et al., 2014), y solo cuando la polaridad del lema estaba entre $-0,5$ y $+0,5$; de este modo nos asegurábamos un claro contenido emocional en la palabra. El análisis así planteado, clasificando en recursos verbales y no verbales positivos o negativos, nos permite enunciar la hipóstesis 7:

H7. Puesto que las emociones con valencia negativa son las que están más presentes, tal y como anunciamos en la hipótesis 2 , los recursos verbales y no verbales con valencia negativa estarán en mayor proporción que los que se presentan con carga positiva.

Como unidad de análisis se tomó cada uno de los tuits publicados; sin embargo, en ocasiones para entender el tuit se ha hecho necesario tener presente los enlaces vinculados o imágenes. La hora de publicación no se menciona, ya que no se considera pertinente y también se evita dar el nombre completo de la cuenta (a

\footnotetext{
${ }^{1}$ Disponible en http://www.lsi.us.es/ fermin/index.php/Datasets
} 
partir de ahora, @username) para evitar cualquier problema que pudiera derivarse, y se transcribe tal y como aparece, a pesar de errores ortográficos o palabras malsonantes.

\section{Resultados}

Para dar respuesta a las preguntas de investigación planteadas, los resultados obtenidos se organizan en 4 partes: emociones y tuits (§ 3.1), estímulos de las emociones (§ 3.2), función de los tuits (§ 3.3) y expresión de las emociones (§ 3.4).

\section{Emociones y tuits}

En términos cuantitativos tal y como se observa en (Tabla 4), cabe destacar que en ambos corpus se produce una actitud emocional semejante: en $\mathrm{C} 1$ con un porcentaje de $86,59 \%$ de tuits emocionales y en C2, con $84,17 \%$ de tuits emocionales. De esta manera se confirma $\mathrm{H} 1$ : hay un mayor número de posts emocionales que informativos.

Tabla 4: Tipo de publicaciones respecto a la emoción

\begin{tabular}{|c|c|c|c|c|c|c|}
\hline & \multicolumn{2}{|l|}{ Corpus } & \multicolumn{2}{|c|}{ C1: \#Maduro } & \multicolumn{2}{|c|}{ C2: @NicolasMaduro } \\
\hline & No tuits & Porcentaje & No tuits & Porcentaje & No tuits & Porcentaje \\
\hline $\begin{array}{l}\text { Sugiere una actitud } \\
\text { emocional }\end{array}$ & 1153 & i & 536 & $86,59 \%$ & 617 & $84,17 \%$ \\
\hline $\begin{array}{l}\text { No contiene actitud } \\
\text { emocional }\end{array}$ & 70 & $5 \%$ & 35 & $5,65 \%$ & 35 & $4,77 \%$ \\
\hline No hay suficiente contexto & 129 & $9,7 \%$ & 48 & $7,75 \%$ & 81 & $11 \%$ \\
\hline Total & 1352 & 100 & 619 & $100 \%$ & 733 & $100 \%$ \\
\hline
\end{tabular}

A continuación varios ejemplos ilustran la carga emocional o ausencia en los tuits:

5. (alegría) @username @username Nunca Fué Tarde Para Oírte,.Enterarme,.Y Ver Tu Mensaje,.Ya De Hastío Contra \#Maduro..\#MiguelBosé..Te Aplaudo...

6. (ira) @ username @NicolasMaduro El ridículo lo hicistes tu Tirano asesino de estudiantes

7. (sin contenido emocional) \#Venezolanos elegir $¥ n$ entre \#laguerraylapaz este 30 de julio: \#Maduro

8. (sin contexto) @username @username @ username tú puedes decirlo directamente a él mencionado @NicolasMaduro

La Tabla 5 muestra la distribución de las emociones. En torno al $61 \%$ de los tuits contienen emociones negativas. 'Ira' $(29,7 \%)$ y 'temor' $(24,5 \%)$ son con mucho las emociones más dominantes. La siguiente emoción con más presencia es orgullo $(13,6)$, de esta manera se confirma -podemos decir- al $75 \%$ la hipótesis 2. Tal y como se había anunciado, las emociones con más presencia siguen siendo 'ira', 'temor' y 'orgullo'; solo 'deseo', que aparecía en tercer lugar en H2, pasa a un quinto puesto en nuestro estudio.

Si comparamos los subcorpus podemos observar que mientras en $\mathrm{C} 1$ hay una clara preeminencia de las emociones negativas $(34,7 \%)$ frente a las positivas $(11,8 \%)$, en C2 vemos que la proporción es 
prácticamente similar, aunque a favor de las positivas (26,9\%) frente a las negativas (26,7\%). Por tanto, $\mathrm{H} 5$ y $\mathrm{H} 6$ quedan refrendada a partir de los datos obtenidos: las emociones con valencia negativa aparecen con mayor frecuencia en $\mathrm{C} 1, \mathrm{y}$ una frecuencia mayor de emociones positivas se concentra en $\mathrm{C}$.

Tabla 5: Relación de emociones y publicaciones

\begin{tabular}{llllll|lll}
\hline & & \multicolumn{3}{l}{ CORPUS } & \multicolumn{3}{l}{ C1: \#Maduro } & \multicolumn{2}{l}{ C2: @NicolasMaduro } \\
\hline Emoción & Descripción & Total & Porcentaje & No tuits & Porcentaje & No tuits & Porcentaje \\
\hline alegría & $1+$ & 130,0 & 11,3 & 46,0 & 4,0 & 84,0 & 7,3 \\
\hline orgullo & $2+$ & 157,0 & 13,6 & 14,0 & 1,2 & 143,0 & 12,4 \\
\hline confianza & $3+$ & 31,0 & 2,7 & 16,0 & 1,4 & 15,0 & 1,3 \\
\hline deseo & $4+$ & 128,0 & 11,1 & 60,0 & 5,2 & 68,0 & 5,9 \\
\hline tristeza & $1-$ & 59,0 & 5,1 & 14,0 & 1,2 & 45,0 & 3,9 \\
\hline vergüienza & $2-$ & 3,0 & 0,3 & 2,0 & 0,2 & 1,0 & 0,1 \\
\hline ira & $2--$ & 342,0 & 29,7 & 190,0 & 16,5 & 152,0 & 13,2 \\
\hline temor & $3-$ & 283,0 & 24,5 & 182,0 & 15,8 & 101,0 & 8,8 \\
\hline sorpresa & $4-$ & 20,0 & 1,7 & 12,0 & 1,0 & 8,0 & 0,7 \\
\hline $\begin{array}{l}\text { Total } \\
\text { emoción }\end{array}$ & & 1153,0 & 100,0 & 536,0 & 46,5 & 617,0 & 53,5 \\
\hline
\end{tabular}

\section{Estímulo de las emociones}

La Figura 2 muestra cómo la frecuencia de los tuits emocionales es claramente mayor en la opción contra el presidente Maduro: $61,9 \%$ contra Maduro frente a 26,4\% a favor. De esta manera se confirma H3: los tuits contrarios a Maduro estarán en mayor proporción que los tuits de los seguidores de Maduro. Respecto al comportamiento de ambos subcorpus, podemos observar cómo hay una clara diferencia entre C1 y C2: mientras en C1 la frecuencia en contra de Maduro $(81,6 \%)$ supera con creces a la frecuencia de tuits emocionales a favor de Maduro $(6,1 \%)$, en el caso de C2 esa proporción prácticamente se equilibra y el resultado es $45,2 \%$ en contra frente a 43,5 . De nuevo $\mathrm{H} 5$ y H6 se cumplen.

A continuación pueden verse algunos ejemplos:

$\mathrm{C} 1$

9. (en contra) @username @username Nunca Fué Tarde Para Oírte,.Enterarme,. Y Ver Tu Mensaje,. Ya De Hastío Contra \#Maduro.. \#MiguelBosé..Te Aplaudo...

10. (a favor) Chévere Presidente PendientesXSIEMPRE Ansiosa X VOTAR \#VenezuelaVotaEn8Dias $X$ PAZ con JUSTICIA - \#Pueblo d \#Bolívar y \#Chávez YA \#Maduro=\#4X4

C2

11. (en contra) Yo desconozco tú "Prostituyente"@NicolasMaduro \#VzlaNoSeRinde \#VzlaResisteEnLaCalle 
12. (a favor) \#FelizLunes todos a votar este 30 de Julio \#VenezuelaVotaEn6Dias @NicolasMaduro https://t.co/RJEY3KFzD2

Figura 2: Polarización de tuits en torno a la figura de Nicolás Maduro

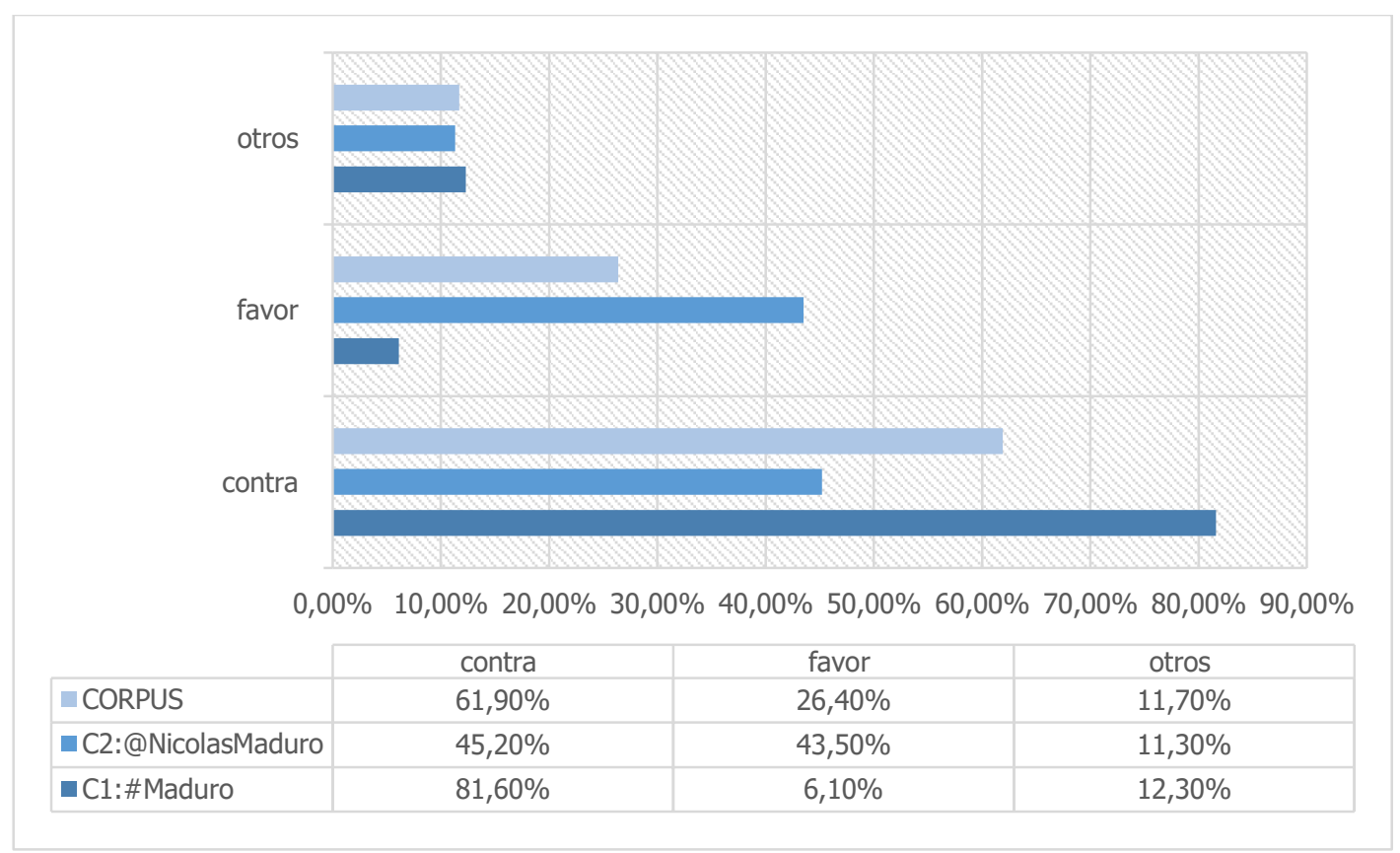

\section{Función de los tuits}

Las respuestas a la pregunta 4 del cuestionario permitió reconocer seis funciones principales entre los tuits publicados: 'denunciar' (21,43)\%, 'ridiculizar' (10,2\%), 'mostrar desacuerdo' (6,4\%) e 'insultar' (6,3\%) entre los tuits contrarios a Maduro, y 'apoyar' $(13,5 \%)$ y 'admirar' $(6,4 \%)$ entre los tuits favorables a Maduro. De las funciones que habían sido anunciadas en H4 solo 'apoyar' y 'admirar' aparecían entre las cuatro con mayor frecuencia en el corpus analizado. Sin embargo, podemos indicar una coincidencia relevante: las funciones anunciadas en $\mathrm{H} 4$ recogen dos funciones positivas hacia una opción y dos negativas, exactamente igual a como ocurre aquí.

Respecto al comportamiento por subcorpus, debemos destacar diferencias significativas: mientras en C1, las funciones principales son denunciar $(14,2)$, ridiculizar $(7,7)$ y mostrar desacuerdo $(6,4)$; en $\mathrm{C} 2$, prevalecen las funciones 'apoyar' $(11,8 \%)$, seguido de 'admirar' $(6,4)$. Con estos resultados debemos volver a $\mathrm{H} 5$ y $\mathrm{H6}$ : en $\mathrm{C} 1$, donde encontramos un mayor número de emociones negativas, resulta lógico que prevalezcan las funciones 'denunciar', 'ridiculizar' y 'mostrar desacuerdo' frente a C2, donde encontramos una frecuencia mayor de emociones positivas, lo que permite que nos encontremos unas funciones distintas, como son 'apoyar' y 'admirar' (a Maduro). Véanse a continuación los siguientes ejemplos:

\section{Denunciar}

13. (denunciar) @NicolasMaduro Nico hijo dale comida al pueblo como quieres gobernar un pueblo con hambre 
14. (ridiculizar) \#PorSiTeLoPerdiste @NicolasMaduro invita al voto con su versión de "Despacito".

15. (apoyar) \#BolivarPrimerConstituyente \#VenezuelaVotaEn6Dias \#VenezuelaCorazondeAmerica \#VamosConLaConstituyente @NicolasMaduro https://t.co/GhtzGxR1Yx

16. (admirar) @NicolasMaduro mi precidente cuente con todo el oriente venezolano no dejaremos mal a nuestro comandante supremo y q no se buelvan loqui

Tabla 6: Funciones de los tuits

\begin{tabular}{lllll}
\hline Tuits & & CORPUS & C1 & C2 \\
& & Porcentaje & Porcentaje & Porcentaje \\
\hline EN CONTRA de Maduro & denunciar & $21,4 \%$ & 14,2 & 7,2 \\
& ridiculizar & $10,2 \%$ & 7,7 & 2,5 \\
& mostrar desacuerdo & 6,4 & 6,4 & 0,0 \\
& insultar & 6,3 & 1,9 & 4,4 \\
& incitar a la acción & 4,3 & 0,2 & 4,1 \\
& informar & 3,7 & 1,9 & 1,8 \\
& desahogarse & 2,4 & 0,7 & 1,8 \\
& amenazar & 1,9 & 1,4 & 0,5 \\
& mostrar hipocresía & 1,4 & 1,4 & 0,0 \\
& provocar & 1,0 & 1,0 & 0,0 \\
& apoyar a la oposición & 0,5 & 0,5 & 0,0 \\
& & & & \\
A FAVOR de Maduro & apoyar & 13,5 & 1,7 & 11,8 \\
& admirar & 6,4 & 0,0 & 6,4 \\
& informar & 5,3 & 0,9 & 4,4 \\
insultar & 0,2 & 0,2 & 0,0 \\
ridiculizar & 0,4 & 0,0 & 0,4 \\
denunciar & 0,2 & 0,0 & 0,2 \\
provocar & 0,2 & 0,0 & 0,2 \\
\hline & & &
\end{tabular}

\section{Expresión de las emociones}

Respecto a cómo se comunican las emociones, la

Tabla 7 muestra, en primer lugar, cómo prevalece el léxico emocional negativo frente al positivo. Del total de tuits analizados, se detectan 1452 palabras pertenecientes al ámbito emocional, de las cuales 759 son negativas $(61,1 \%)$ frente a 483 positivas $(38,9 \%)$. De este modo se confirma la hipótesis 7: los recursos verbales y no verbales con valencia negativa estarán en mayor proporción que los que se presentan con carga positiva.

Si atendemos a la comparación de ambos subcorpus, cabe llamar la atención sobre varios hechos. En primer lugar, en $\mathrm{C} 1$, prevalecen sustantivos y adjetivos negativos (38,2\%, negativos, frente a 13,5\%, positivos) y, sin embargo, en $\mathrm{C} 2$ son los lexemas con carga positiva los que tienen más presencia (25,4\%, positivos frente a 22,9, negativos). Este hecho lingüístico viene a confirmar nuevamente el comportamiento emocional 
defendido en las hipótesis 5 y 6: H5. En las publicaciones con el hashtag \#Maduro prevalecen las emociones negativas. H6. En las publicaciones con la mención @NicolasMaduro prevalecen las emociones positivas. A continuación pueden verse algunos ejemplos que ilustran estas elecciones:

17. (ira) \#Maduro merece la muerte... No hay piedad para esa bestia: Dios, un criminal que mata tanta gente del pueblo $d$ Vene... twitter.com/i/web/status/8...

18. (alegría) Con esta alegría, vamos rumbo a la Constituyente, rumbo a la victoria! @NicolasMaduro @username @username...

En segundo lugar y en referencia a las expresiones de tono más bronco (insultos, trolling), podemos decir que el comportamiento es desigual: los insultos son preferidos en $\mathrm{C} 1 ; 66,4 \%$ del total de insultos encontramos en C1 frente a 33,6\% en C2. Y en el caso de los trolling ocurre al revés: un 35\% de los trolling están en $\mathrm{C} 1$ frente al $65 \%$ restante que se encuentra en C2. A continuación presentamos algunos ejemplos:

Insultos

19. @Irene_Montero_eres una hjija de puta por ensuciar los DDHH cuando @ahorapodemos recibe dienro manchado de sangre... twitter.com/i/web/status/8...

20. @juanc_34 \#MeCagoEnMaduro en la Madre de \#Maduro y en la Madre de cuanto \#Chavista hijo de putas exista... twitter.com/i/web/status/8...

Trolling

21. \#guebo \#chicos \#hard \#gayporn \#TopGayXXX \#Tripemx \#sex \#hard \#paja \#fetiche \#maduro \#corridas \#paja https://t.co/JksBbzuiLw

22. Este maduro me puede \#Pete \#Maduro \#Leche \#Morbo. Dale me gusta y RT https://t.co/6yz95ro3Hu

Sin embargo, respecto a las preguntas retóricas e ironía, se observa un patrón muy similar en ambos subcorpus. Veamos los siguientes ejemplos:

Ironía

23. VENEZUELA. La "pacífica" oposición venezolana ya ha quemado vivas a 23 personas. @dcabellor @username @username @username @NicolasMaduro

24. \#maduro tengo un juego para ti : Muere periodista en Maracaibo tras realizar reto "Ballena Azul".

Pregunta retórica

25. (pregunta retórica) Es eso lo que hace @NicolasMaduro ? Quería el Libertador tanta represión, asesinatos y un país tan desigual? ...

26. ¿Quién realmente da las órdenes al retardado \#Maduro? \#Cuba? \#Rusia? \#Irán? \#SOSVenezuela \#Democracia \#NoAlComunismo 
Por último, los mecanismos de intensificación de las emociones (repetición de emoticonos, letras, sonidos, mayúsculas) ponen de manifiesto que en ambos subcorpus existe una necesidad clara de expresar las emociones con una gran intensidad. Véanse los ejemplos siguientes:

27. (alegría) @VTVcanal8 @NicolasMaduro $\square \square \square \square \square \square \square \square$ si va esta constituyente.Dios les bendiga gracias.por los niño niñas chave vive

28. (alegría) @username @NicolasMaduro y preparense xq ahora es que hay chavistasssssssss y pa ratoooooo. 30] a votar todos a votar y punto....y punto

29. (ira) @NicolasMaduro CANDIDATOS MALANDROS, MUERTOS DE HAMBRES ESPERANDO CLAP, COMUNISTAS SON LOS DEL MABURRO ESTAS INCLUIDO EN ESE PAQUETE

Tabla 7: Modos de expresión de las emociones

\begin{tabular}{|l|l|l|}
\hline & C1: \#Maduro & C2: @NicolasMaduro \\
\hline Léxico emocional negativo & 474 & 285 \\
\hline Léxico emocional positivo & 168 & 315 \\
\hline Insultos & 140 & 71 \\
\hline Trolling & 14 & 26 \\
\hline Ironía & 24 & 28 \\
\hline Preguntas retóricas & 34 & 37 \\
\hline Emoticonos & 31 & 37 \\
\hline Repetición de letras & 6 & 16 \\
\hline Repetición de signos (i, !!, i!!!, i!!!!) & 59 & 17 \\
\hline Mayúsculas & 16 & 34 \\
\hline
\end{tabular}

\section{Discusión y conclusiones}

A partir de los resultados obtenidos, en primer lugar, encontramos que Twitter es usado como una plataforma para la deliberación política. La segunda conclusión que se deduce es la importancia clave que adquieren las emociones en el discurso metapolítico. Este discurso emocional emerge espontáneamente del ciudadano ante las contradicciones, controversias y conflictos vividos en un país, como es el caso de Venezuela durante el gobierno de Nicolás Maduro. Por ello, los tuits se convierten en la herramienta idónea para compartir las emociones 'ira' y 'temor', que son las emociones que con más frecuencia aparecen en el corpus seleccionado, en el $54,2 \%$ del total de tuits analizados.

Con respecto a nuestro tercer objetivo, analizar el estímulo de la emoción, recordaremos, en primer lugar, que en los tuits analizados existía una clara polarización entre los partidarios del Presidente Maduro y la gran coalición opositora contraria a Maduro (MUD). En esta polarización, "contrarios frente adeptos" a Maduro, es significativo y se debe destacar cómo más de un $80 \%$ de tuits analizados son contrarios a Maduro. Por tanto, es digno de reseñar cómo esta plataforma se convierte en un canal de lucha, protesta y reivindicación del votante minoritario que siente que sus derechos se hallan vulnerados. De nuevo constatamos el activismo político, que se ha visto en protestas anteriores, donde se comparten emociones, 
fines y herramientas (Castells, 2012). Respecto a la comparación entre ambos subcorpus, cabe destacar cómo los subcorpus $\mathrm{C} 1$ y C2 actúan de un modo distinto, respecto a la polarización. Puesto que la mención es entendida como un vocativo y automáticamente se muestra en el "timeline" de la persona mencionada, nos encontramos que en el corpus 2 (@NicolasMaduro) emergen los tuits favorables a Maduro y, como consecuencia, esta opción permite que veamos más emociones positivas: los fieles a Maduro prefieren elegir la mención, sabedores de que aparece en la cuenta del Presidente, hecho que les genera 'orgullo' y 'alegría'. Por el contrario, el trend topic \#Maduro (C1) se entiende como una vía neutra para expresar emociones en contra de Nicolás Maduro, sin la presión de sentirse tan controlados; este hecho explica que en este subcorpus el porcentaje de emociones negativas alcance un $34,7 \%$ frente a tan solo $11,8 \%$ de positivas. Respecto a la función de los tuits, se advierte que esta viene determinada no por los subcorpus en los que se ubican, sino por la polarización, su postura "hacia o contra" Maduro. Con los tuits emocionalmente negativos al presidente Maduro se busca 'denunciar' y 'ridiculizar, fundamentalmente; con los tuits favorables a Maduro, 'apoyar' y 'admirar' al Presidente. Podemos decir que los tuits tanto a favor como en contra tiene un claro carácter instrumental, una función persuasiva, puesto que ellos, excepción de los tuits de "desahogo", usan Twitter para promover uno de los dos partidos, bien tuiteando abiertamente sobre qué opción es la mejor ('apoyar', 'admirar)', bien indirectamente presentado las "buenas" o "malas" acciones de un bando o de otro ('denunciar', 'ridiculizar', 'insultar', 'mostrar hipocresía').

Por último el análisis de los recursos verbales y no verbales que expresan la valencia de las emociones y/o su intensidad sugiere un claro desequilibrio a favor de los recursos emocionales negativos. Este modo de proceder afecta también a las expresiones y estilos seleccionados: más número de palabras con carga negativa (palabras emocionales e insultos) en C1 que en C2. Sin embargo, en C2 cabe destacar cómo emergen un mayor número de trolling; este hecho no debe chocar, si pensamos que la procacidad es mayor si se le menciona directamente a la persona implicada.

En suma, este análisis emocional viene a enriquecer la comprensión del uso de Twitter en el ámbito político. Deseamos contribuir a la investigación sobre el comportamiento político del ciudadano en las redes sociales, al acercarnos a su participación tomando como referencia las emociones en los tuits durante la campaña electoral. Consideramos que los resultados obtenidos deberán ser tenidos en cuenta en futuros estudios que centren la atención en el comportamiento emocional expresado en Twitter, especialmente en campañas electorales marcadas por una clara polarización. Sin embargo, somos conscientes de que este estudio requiere de posteriores análisis. Estos análisis podrían verse completado con análisis de sentimientos automatizados que incluyera los 6 días monitorizados; la combinación del método manual y el automatizado nos permitiría una comprensión más profunda del comportamiento emocional del ciudadano en elecciones especialmente polarizadas y complicadas ${ }^{2}$, al mismo tiempo que podría ayudar en la mejora de los métodos de análisis automatizados.

\footnotetext{
${ }^{2}$ El autor declara que no existe ningún potencial conflicto de intereses con respecto a la investigación y/o publicación de esta investigación.
} 


\section{Referencias}

Albertson, B., \& Gadarian, S. K. (2015). Anxious politics: Democratic citizenship in a threatening world. Cambridge: Cambridge University Press.

Aourag, M \& A. Alexander. (2011). The Egyptian experience: sense and non-sense of the Internet revolution. International Journal of Communication, 5, 1344-1358. Disponible en: http://ijoc.org/index.php/ijoc/article/view/1191/610

Baker, J. (1987). The role of environment in marketing service: The consumer perspective. En J. A, Czepid et al., eds., The Service Challenge: Integrating for Competitive Advantage (pp. 79-84). Chicago, IL: American Marketing Association.

Bednarek, M. (2008). Semantic preference and semantic prosody re-examined. Corpus Linguistics and Linguistic Theory, 4(2), 119-139. DOI 10.1515/CLLT.2008.006

Bonilla, Y., \& Rosa, J. (2015). \# Ferguson: Digital protest, hashtag ethnography, and the racial politics of social media in the United States. American Ethnologist, 42(1), 4-17. https://doi.org/10.1111/amet.12112

Borge-Holthoefer, J., Magdy, W., Darwish, K., \& Weber, I. (2015, February). Content and network dynamics behind Egyptian political polarization on Twitter. In Proceedings of the 18th ACM Conference on Computer Supported Cooperative Work \& Social Computing (pp. 700-711). ACM. Disponible en: https://arxiv.org/pdf/1410.3097.pdf

Brader, T., \& Corrigan, B. (2006). How the emotional tenor of ad campaigns affects political participation. In Annual meeting of the American Political Science Association, Philadelphia. Disponible en: https://goo.gl/aFLhFd

Braun, M. (2015). Emotion and language-When and how comes emotion into words? Comment on "The quartet theory of human emotions: An integrative and neurofunctional model" by S. Koelsch et al. Koelsch et al. Phys. Life Rev, 13, 36-37.

Brody, S., \& Diakopoulos, N. (2011, July). Coooooooooooooooll||IIIIIIIII!!!!!!!!!!!!!!!: using word lengthening to detect sentiment in microblogs. In Proceedings of the conference on empirical methods in natural language processing (pp. 562-570). Association for Computational Linguistics. Disponible en: http://www.anthology.aclweb.org/D/D11/D11-1052.pdf

Brosch, T., Scherer, K. R., Grandjean, D. M., \& Sander, D. (2013). The impact of emotion on perception, attention, memory, and decision-making. Swiss medical weekly, 143, w13786. DOI: $10.4414 /$ smw.2013.13786

Bruns, A., \& Burgess, J. E. (2011, August). The use of Twitter hashtags in the formation of ad hoc publics. In Proceedings of the 6th European Consortium for Political Research (ECPR) General Conference 2011. Disponible en: https://goo.gl/fkAVRH

Caffi, C., \& Janney, R. W. (1994). Toward a pragmatics of emotive communication. Journal of pragmatics, 22(3), 325-373. http://dx.doi.org/10.1016/0378-2166(94)90115-5

Calero Vaquera, M. L., \& Vigara Tauste, A. (2014). El discurso del whatsapp: entre el Messenger y el Sms. Oralia, $17,87-116$ 
Caragea, C., McNeese, N., Jaiswal, A., Traylor, G., Kim, H. W., Mitra, P., ... \& Yen, J. (2011, May). Classifying text messages for the haiti earthquake. In Proceedings of the 8th international conference on information systems for crisis response and management (ISCRAM2011). Disponible en: http://citeseerx.ist.psu.edu/viewdoc/download?doi=10.1.1.370.6804\&rep=rep1\&type=pdf

Castells, M. (2009). Comunicación y Poder. Madrid: Alianza Editorial.

Castells, M. (2012). Redes de indignación y esperanza. Madrid: Alianza Editorial.

Cha, M., Haddadi, H., Benevenuto, F., \& Gummadi, P. K. (2010). Measuring user influence in twitter: The million follower fallacy. Icwsm, 10(10-17), 30ss. Disponible en: https://goo.gl/1798fP

Collier, N., Son, N. T., \& Nguyen, N. M. (2011). OMG U got flu? Analysis of shared health messages for biosurveillance. Journal of biomedical semantics, 2(5), S9. Disponible en: https://link.springer.com/article/10.1186\%2F2041-1480-2-S5-S9

Costanza-Chock, S. (2012). Mic check! Media cultures and the Occupy movement. Social movement studies, 11(3-4), 375-385. http://dx.doi.org/10.1080/14742837.2012.710746

Cruz, F. L., Troyan, J. A., Pontes, B., \& Ortega, F. J. (2014). ML-SentiCon: Un lexicón multilingüe de polaridades semánticas a nivel de lemas. Procesamiento del Lenguaje Natural, (53). Disponible en: http://hdl.handle.net/10045/40031

Damasio, A. R. (1999). How the brain creates the mind. Scientific American, 281(6), 112-117. Disponible en: https://goo.gl/fZ35pm

Dang-Xuan, L., Stieglitz, S., Wladarsch, J., \& Neuberger, C. (2013). An investigation of influentials and the role of sentiment in political communication on Twitter during election periods. Information, Communication \& Society, 16(5), 795-825. https://doi.org/10.1080/1369118X.2013.783608

De León, J. \& Jones, C. (2011). Tunisia and Egypt: Unrest and Revolution. NY: Nova Science Publishers, Inc.

Dodds, P. S., Harris, K. D., Kloumann, I. M., Bliss, C. A., \& Danforth, C. M. (2011). Temporal patterns of happiness and information in a global social network: Hedonometrics and Twitter. PloS one, 6(12), e26752. https://doi.org/10.1371/journal.pone.0026752

Foolen, A. (2011). Pragmatic markers in a sociopragmatic perspective. En A. Gisle and K. Aijmer (eds.). Pragmatics of society, pp. 217-282. Berlin; Boston: De Gruyter Mouton.

Foolen, A. (2012). The relevance of emotion for language and linguistics. En A. Foolen., U.M. Lüdtke, T.P. Racine y J. Zlatev (Eds.). Moving ourselves, moving others: motion and emotion in intersubjectivity, consciousness and language (Vol. 6), 349-369. Amsterdam: John Benjamins Publishing.

Garwood-Gowers, A. (2013). The responsibility to protect and the Arab Spring: Libya as the exception, Syria as the norm. UNSWL, 36, 594ss. Disponible en: https://eprints.qut.edu.au/61708/

Gibbs, R. (2000). Irony in talk among friends. Metaphor and Symbol, 15, 5-27. Disponible en: http://www.tandfonline.com/loi/hmet20

Gildea, D., \& Jurafsky, D. (2002). Automatic labeling of semantic roles. Computational linguistics, 28(3), 245-288. doi>10.1162/089120102760275983

Go, A., Bhayani, R., \& Huang, L. (2009). Twitter sentiment classification using distant supervision. CS224N Project Report, Stanford, 1(12). http://www.yuefly.com/Public/Files/2017-0307/58beb0822faef.pdf 
Ghosh, A., Li, G., Veale, T., Rosso, P., Shutova, E., Barnden, J., \& Reyes, A. (2015). Semeval-2015 task 11: Sentiment analysis of figurative language in twitter. In Proceedings of the 9th International Workshop on Semantic Evaluation (SemEval 2015) (pp. 470-478). Disponible en: http://alt.qcri.org/semeval2015/cdrom/pdf/SemEval080.pdf

Graham, S., \& Hewitt, L. (2013). Getting off the ground: On the politics of urban verticality. Progress in Human Geography, 37(1), 72-92. DOI: 10.1177/0309132512443147

Grillo, M. C. (2017). Nationalist Politics: The Role of Predispositions and Emotions. Why Irrational Politics Appeals: Understanding the Allure of Trump. Santa Barbara, California: ABC-CLIO, LLC.

Grimmer, J., \& Stewart, B. M. (2013). Text as data: The promise and pitfalls of automatic content analysis methods for political texts. Political analysis, 21(3), 267-297. doi:10.1093/pan/mps028

Grossman, L. 1995. The Electronic Republic. New York: Penguin Books.

Guerrero, M. P. (2014). Anuncios de prensa escrita alemana: Desarrollo temático, locución e implicación emocional. Anuari de filologia. Estudis de lingüística, (4), 205-206. Disponible en: http://revistes.ub.edu/index.php/AFEL/article/viewFile/11151/13916

Hopkinson, C. (2013). Trolling In Online Discussions: From Provocation To Community-Building. Brno studies in English, 39(1). http://hdl.handle.net/11222.digilib/129149

Huddy, L., Cassese, E., \& Lizotte, M. K. (2008). Gender, public opinion, and political reasoning. Political women and American democracy, 31-49. doi:10.1017/S1743923X14000567

Hosch-Dayican, B., Amrit, C., Aarts, K., \& Dassen, A. (2016). How do online citizens persuade fellow voters? Using Twitter during the 2012 Dutch parliamentary election campaign. Social science computer review, 34(2), 135-152. DOI: 10.1177/0894439314558200

Hutchings, V. L., Valentino, N. A., Philpot, T. S., \& White, I. K. (2006). Racial cues in campaign news: The effects of candidate strategies on group activation and political attentiveness among African Americans. In Redlawsk (ed). Feeling politics (pp. 165-186). Palgrave Macmillan, New York.

Iriarte, D. (28 de julio de 2017). Elecciones a la Asamblea Constituyente: claves de un proceso crucial para Venezuela. El Confidencial. Disponible en: https://goo.gl/dbmPcg

Kilgarriff, A., Baisa, V., Bušta, J., Jakubíček, M., Kováŕ, V., Michelfeit, J., ... \& Suchomel, V. (2014). The Sketch Engine: ten years on. Lexicography, 1(1), 7-36. Disponible en: https://link.springer.com/article/10.1007\%2Fs40607-014-0009-9

Kreuz, R. J. (1996). The use of verbal irony: Cues and constraints. En J. S Mio y A. Katz, (Editores), Metaphor: Implications and Applications, Mahwah, Lawrence Erlbaum Associates, New Jersey: 23-38.

Kuklinski, J. H., Luskin, R. C., \& Bolland, J. (1991). Where is the schema? Going beyond the " $\mathrm{S}$ " word in political psychology. American Political Science Review, 85(4), 1341-1380. DOI: 10.2307/1963949

Lassen, D. S., \& Brown, A. R. (2011). Twitter: The electoral connection?. Social Science Computer Review, 29(4), 419-436. DOI: 10.1177/0894439310382749

Lee, C., \& Kahle, L. (2016). The Linguistics of Social Media: Communication of Emotions and Values in Sport. Sport Marketing Quarterly, 25(4), 201ss. Disponible en: http://www.fitpublishing.com/articles/linguistics-social-media-communication-emotions-andvalues-sport 
Liu, B. (2012). Sentiment analysis and opinion mining. Synthesis lectures on human language technologies, 5(1), 1-167. Disponible en: https://www.cs.uic.edu/ liub/FBS/SentimentAnalysisand-OpinionMining.pdf

Lodge, M., \& Taber, C. S. (2005). The automaticity of affect for political leaders, groups, and issues: An experimental test of the hot cognition hypothesis. Political Psychology, 26(3), 455-482. https://doi.org/10.1111/j.1467-9221.2005.00426.x

Marcus, G. E. (2003). The Psychology of Emotion and Politics. In D. Sears, L. Huddy, and R. Jervis (eds.). Oxford Handbook of Political Psychology, New York: Oxford University Press, 182-221.

Marcus, G. E., Neuman, W. R., \& MacKuen, M. (2000). Affective intelligence and political judgment. Chicago: University of Chicago Press.

Meza, A. (17 de julio de 2017). La oposición venezolana asegura que logró casi 7,2 millones de votos en la consulta contra Maduro. El País. Disponible en: https://goo.gl/VSMEBw

Mohammad, S. M., Zhu, X., Kiritchenko, S., \& Martin, J. (2015). Sentiment, emotion, purpose, and style in electoral tweets. Information Processing \& Management, 51(4), 480-499. Disponible en: https://dl.acm.org/citation.cfm?id=2793724.2793929

Mossberger, K., Tolbert, C. J., \& McNeal, R. (2008). Digital citizenship: The Internet, society, and participation. Cambridge, MA: MIT Press.

Morales, A. J., Borondo, J., Losada, J. C., \& Benito, R. M. (2015). Measuring political polarization: Twitter shows the two sides of Venezuela. Chaos: An Interdisciplinary Journal of Nonlinear Science, 25(3), 033114. https://doi.org/10.1063/1.4913758

Nabel, L. C. T. (2015). ¿Quién programa las redes sociales en Internet? El caso de Twiter en el movimiento\# Yosoy132 México. Revista Internacional de Sociología, 73(2), 010. https://doi.org/10.3989/ris.2013.05.29

O'Connor, B., Balasubramanyan, R., Routledge, B. R., \& Smith, N. A. (2010). From tweets to polls: Linking text sentiment to public opinion time series. ICWSM, 11(122-129), 1-2. Disponible en: https://goo.gl/TEZBLW

Pang, B., \& Lee, L. (2008). Opinion mining and sentiment analysis. Foundations and Trends $®$ in Information Retrieval, 2(1-2), 1-135. Disponible en:

Persson, G. (2017). Love, Affiliation, and Emotional Recognition in\# kämpamalmö:-The Social Role of Emotional Language in Twitter Discourse. Social Media+ Society, 3(1), 1-11. doi/pdf/10.1177/2056305117696522

Rheingold, H. 1993. Homesteading on the Electronic Frontier. Reading, MA: Addison-Wesley.

Rossi, L., \& Magnani, M. (2012, June). Conversation Practices and Network Structure in Twitter. In ICWSM. Disponible

en: https://pdfs.semanticscholar.org/a3bc/a5dc6daee09b4a0b715a1d6b23c904ddcda3.pdf

Russell, J. A. (2009). Emotion, core affect, and psychological construction. Cognition and Emotion, 23(7), 1259-1283. DOI: 10.1080/02699930902809375

Scherer, K. R., Shuman V., Fontaine J.R.J. \& Soriano C. (2013). Components of emotional meaning: A sourcebook, 281-298. Oxford: Oxford University Press.

Scheff, T. (2015). Toward defining basic emotions. Qualitative Inquiry, 21(2), 111-121. DOI: $10.1177 / 1077800414550462$ 
Smith, C. A., \& Ellsworth, P. C. (1985). Patterns of cognitive appraisal in emotion. Journal of personality and social psychology, 48(4), 813. DOI:10.1037//0022-3514.48.4.813

Stieglitz, S \& Dang-Xuan L. (2013). Emotions and information diffusion in social media-sentiment of microblogs and sharing behavior. Journal of Management Information Systems, 29 (4), 217-248. DOI: $10.2753 / M I S 0742-1222290408$

Strapparava, C., \& Mihalcea, R. (2007, June). Semeval-2007 task 14: Affective text. In Proceedings of the 4th International Workshop on Semantic Evaluations (70-74). Association for Computational Linguistics. Disponible en: https://goo.gl/B3uTd1

Tam Cho, W. K., Gimpel, J. G., \& Hui, I. S. (2013). Voter migration and the geographic sorting of the American electorate. Annals of the Association of American Geographers, 103(4), 856-870. https://doi.org/10.1080/00045608.2012.720229

Tarrow, S. (10 de octubre de 2011). Why Occupy Wall Street is not a tea party of the left. Foreing Affairs. Disponible en: https://goo.gl/GeDZjc

Valentino, N. A., Brader, T., Groenendyk, E. W., Gregorowicz, K., \& Hutchings, V. L. (2011). Election night's alright for fighting: The role of emotions in political participation. The Journal of Politics, 73(1), 156-170. DOI: $10.1017 /$ S0022381610000939

Vergeer, M., Hermans, L., \& Sams, S. (2013). Online social networks and micro-blogging in political campaigning: The exploration of a new campaign tool and a new campaign style. Party Politics, 19(3), 477-501. DOI: 10.1177/1354068811407580

Vilares, D., \& Alonso Pardo, M. Á. (2016). A review on political analysis and social media. Procesamiento del lenguaje natural, 56, 13-23. Disponible en: http://hdl.handle.net/10045/53557

Zajonc, R. B. (1980). Feeling and thinking: Preferences need no inferences. American psychologist, 35(2), 151. http://dx.doi.org/10.1037/0003-066X.35.2.151

Zappavigna, M. (2012). Discourse of Twitter and social media: How we use language to create affiliation on the web (Vol. 6). Londres: Continuum. doi: 10.11139/cj.31.3.412-414

Zeitzoff, T. (2016). Does social media influence conflict? Evidence from the 2012 Gaza Conflict. Journal of Conflict Resolution, p. 0022002716650925 . DOI: $10.1177 / 0022002716650925$ 